\title{
ULTRAVIOLET PHOTOMETRY OF STARS FROM OSO II
}

\author{
KENNETH L. HALLAM \\ Goddard Space Flight Center, Greenbelt, Md., U.S.A.
}

\begin{abstract}
Discussion
Abstract. Ultraviolet stellar fluxes from 1500 to $3200 \AA$ were from February through August 1965 on OSO II. A $15-\mathrm{cm}$ diameter Gregorian telescope with a stepped grating spectrophotometer provided flux measurements in ten adjacent $180 \AA$ wide band passes.

By comparing fluxes of stars showing B-U color excesses smaller than 0.19 and larger than 0.26 , an ultraviolet extinction curve has been derived, which agrees with others which have been published as far as the magnitude effect is concerned, but the shape differs somewhat, the slope at $1 / \lambda=3.8 \mu^{-1}$ being somewhat greater than at $1 / \lambda=5.0 \mu^{-1}$.

If the stars' fluxes are corrected for reddening, it is found that there is a good agreement between observation and models for stars earlier than B3, but that many of the later type stars have a residual apparently intrinsic reddening.

Greenberg: Has Hallam investigated the possibility that apparently anomalous properties of the later type stars may be due to the fact that the extinction curve derived from the early type stars is not applicable to the later types? The early-type stars could modify the neighbouring interstellar medium and since the stars which have been studied so far for extinction are relatively nearby (total extinction rather small) a significant proportion of the overall extinction could be of local origin.

Roman: I doubt it. However, I would be very surprised if these stars are sufficiently reddened to explain the problem in this way.
\end{abstract}

